\title{
Optofluidic Distributed Feedback Dye Laser
}

\author{
Zhenyu Li, Zhaoyu Zhang, Teresa Emery, Axel Scherer, and Demetri Psaltis \\ Department of Electrical Engineering, California Institute of Technology, Pasadena, California 91125 \\ zhenyu@caltech.edu
}

\begin{abstract}
We demonstrated an optofluidic distributed feedback (DFB) dye laser on a poly(dimethylsiloxane) (PDMS) chip. Single-mode operation was obtained with $0.21 \mathrm{~nm}$ linewidth. We achieved nearly $60 \mathrm{~nm}$ continuously tunable output by mechanically varying the grating period.

C)2006 Optical Society of America

OCIS codes: (140.2050) Dye lasers; (140.3490) Lasers, distributed-feedback; (140.3570) Lasers, single-mode; (140.3600) Lasers, tunable; (230.4000) Microstructure fabrication; (230.7380) Waveguides, channeled; (130.0130) Integrated optics
\end{abstract}

On-chip liquid dye lasers are ideal coherent light sources for 'lab-on-a-chip' systems. Recently, several groups have demonstrated such dye lasers using different materials and laser cavities [1-3]. Here we show, to our knowledge, the first DFB liquid dye laser on a PDMS chip [4]. We also study the mechanical tunability of such lasers.

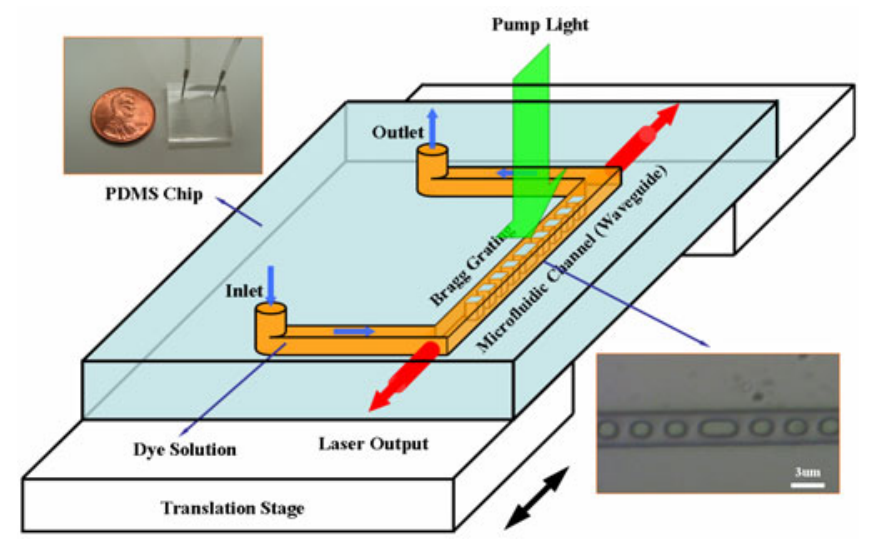

Fig.1. Schematic diagram of an optofluidic DFB dye laser chip. The upper inset shows an actual PDMS laser chip. The lower inset is an optical micrograph of the central phase-shifted region of the laser cavity. The scale bar is $3 u m$.

As shown in Fig. 1, the optofluidic DFB dye laser was fabricated on a monolithic PDMS chip using replica molding soft lithography as described in $[4,5]$. A microfluidic channel when filled with liquid of higher refractive index than that of PDMS (1.406, GE RTV615) acts as a channel waveguide. When the channel dimensions are $2 \mathrm{um} \times 5 \mathrm{um}$ and the index contrast is less than 0.003 , the waveguide supports only the fundamental $\mathrm{E}_{11}$ modes. The distributed feedback is provided by the periodic PDMS posts inside the channel with $3 \mathrm{um}$ period, which form a 4mm long 15 thorder Bragg grating at wavelength around $565 \mathrm{~nm}$. A $15 \pi$ phase shift is introduced at the center to ensure single frequency operation. The gain medium is a $2 \mathrm{mM}$ solution of Rhodamine $6 \mathrm{G}(\mathrm{Rh} 6 \mathrm{G})$ or Rhodamine $101(\mathrm{Rh} 101)$ in a methanol and ethylene glycol mixture with refractive index of 1.409. The Bragg wavelength of a DFB laser is determined by the condition

$$
m \lambda_{m}=2 n_{\text {eff }} \Lambda
$$

where $\lambda_{m}$ is the $m$ th order resonant wavelength, $n_{e f f}$ is the effective index of the guided mode and $\Lambda$ is the grating period. Given $\Lambda=3 \mathrm{um}$ and $n_{\text {eff }}=1.407$, the 15 th resonant wavelength and the free spectral range (FSR) are $563 \mathrm{~nm}$ and $40.2 \mathrm{~nm}$ respectively. Furthermore, the even order resonances are absent when a $50 \%$ duty-cycle grating is used. The resulting extremely large $F S R$ ensures a single resonance inside the gain spectrum.

The lasing wavelength can be tuned by changing $n_{\text {eff }}, \Lambda$ or $m$. Due to the low Young's modulus of PDMS ( $\sim 750 \mathrm{kPa}[6])$, the grating period $\Lambda$ can be easily varied by stretching or compressing the chip. The grating order $m$ can be chosen by using different dye molecules which cover different spectral regions.

The laser chip was optically pumped with 6ns Q-switched Nd:YAG laser pulses of 532nm wavelength, focused to a $\sim 100 \mu \mathrm{m}$ wide stripe aligned with the microfluidic channel. A typical single mode lasing spectrum is shown in Fig. 2 where the lasing wavelength is $567.3 \mathrm{~nm}$, close to the predicted $563 \mathrm{~nm}$. The measured linewidth is $0.21 \mathrm{~nm}$. A 


\section{CFJ1.pdf}

plot of light output vs. light input curve is shown as the inset $\mathbf{B}$ of Fig. 2. The threshold pump fluence is estimated to be $\sim 0.8 \mathrm{~mJ} / \mathrm{cm}^{2}$.

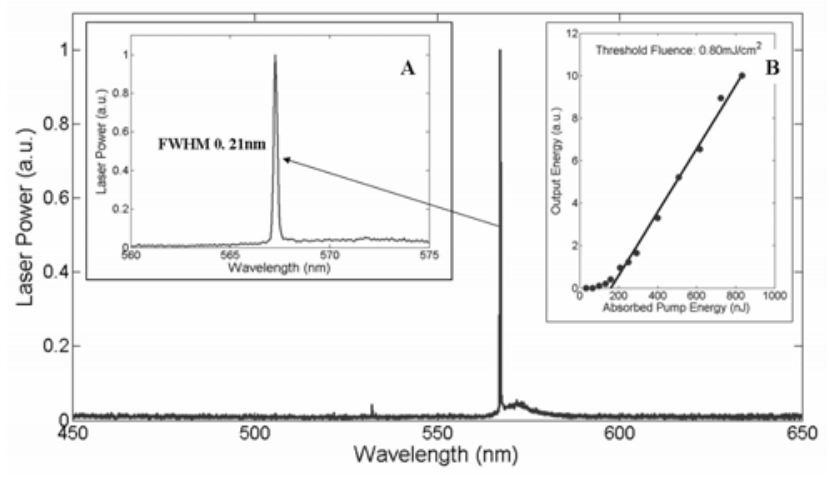

Fig.2. Optofluidic DFB dye laser spectrum. The inset B shows the output energy vs. the absorbed pump energy curve.
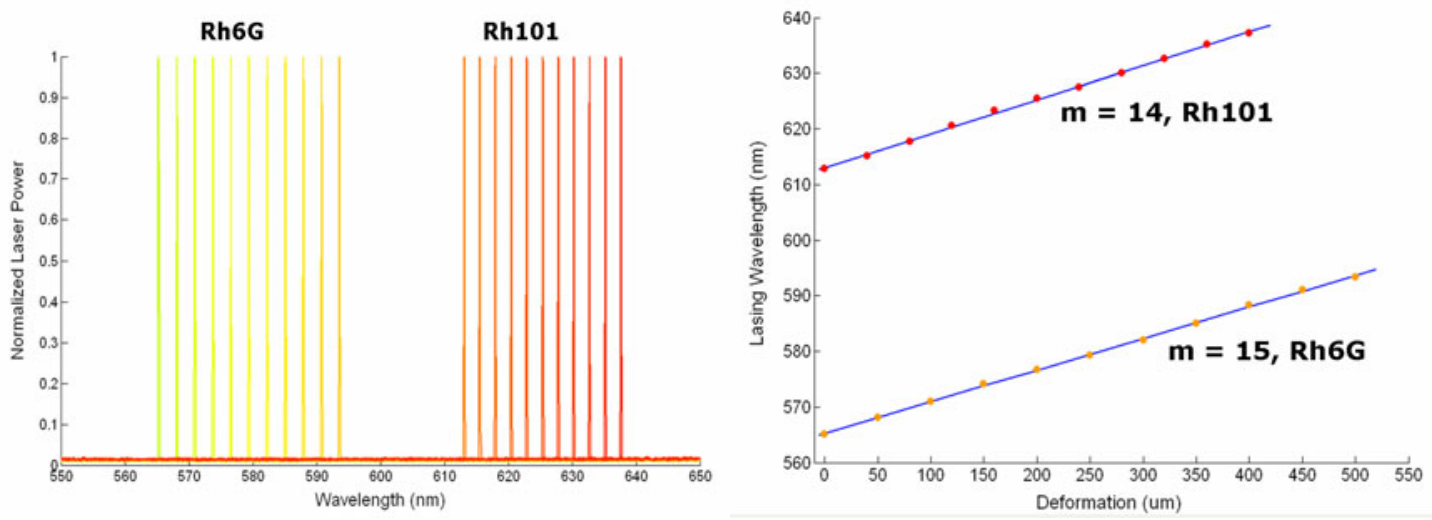

Fig.3. Left: normalized laser output of the mechanically tunable optofluidic DFB dye laser. Different peaks correspond to different grating periods. Right: lasing wavelength versus the measured chip deformation.

We also achieved continuously tunable output by mechanically varying the grating period. Fig. 3 . shows that the achieved single mode tuning range for Rh6G is from $565 \mathrm{~nm}$ to $594 \mathrm{~nm}$ and from $613 \mathrm{~nm}$ to $638 \mathrm{~nm}$ for Rh101. Throughout the tuning range, stable single-mode operation was maintained. Because of the multiple spectral resonances supported by the higher order grating, this laser can provide tunable output covering from near UV to near IR spectral region.

We have demonstrated the first continuously tunable single mode DFB liquid dye laser on a PDMS chip. The fabrication and operation of the laser chip is fully compatible with PDMS based microfluidics technology [7]. Such lasers may enable the implementation of spectrometers, biosensors and imaging systems on chip.

\section{References}

[1] B. Helbo, A. Kristensen, and A. Menon, “A micro-cavity fluidic dye laser,” J. Micromech. Microeng. 13, 307-311 (2003).

[2] D.V. Vezenov, B.T. Mayers, R.S. Conroy, G.M. Witesides, P.T. Snee, Y. Chan, D.G. Nocera, and M.G. Bawendi, “A low-threshold, highefficiency microfluidic waveguide laser," J. AM. Chem. Soc. 127, 8952-8953 (2005).

[3] J.C. Galas, J. Torres, M. Belotti, and Q. Kou, "Microfluidic tunable dye laser with integrated mixer and ring resonator," App. Phys. Lett. 86, 264101 (2005).

[4] Z. Li, Z. Zhang, T. Emery, A. Scherer and D. Psaltis, "Single mode optofluidic distributed feedback dye laser", submitted (2005).

[5] Y.N. Xia and G.M. Whitesides, "Soft lithography,” Annu. Rev. Mater. Sci. 28, 153-184 (1998).

[6] J.C. McDonald and G.M. Whitesides, "Poly(dimethylsiloxane) as a material for fabricating microfluidic devices," Acc. Chem. Res. 35, 491499 (2002).

[7] M.A. Unger, H.P. Chou, T. Thorsen, A. Scherer, S.R. Quake, "Monolithic microfabricated valves and pumps by multilayer soft lithography," Science, 288, 113-116 (2000).

\section{Acknowledgments}

This research was supported by the DARPA center for optofluidic integration. 